\title{
Politische Verantwortung in der Marktwirtschaft Zur Übertragung von Citizenship-Konzepten auf Konsumenten und Unternehmen
}

\author{
VERONIKA KNEIP
}

Mit der Verwendung des Bürgerbegriffs für Konsumenten und Unternehmen erfolgt eine Verschränkung von Wirtschaftssphäre und politischer Arena, die in einem Spannungsverhältnis zu bestehenden Entwürfen von (Staats-)Bürgerschaft steht. Gegenstand des Beitrags ist es, diese Neukonfigurationen zu hinterfragen und dabei sowohl analytische Potenziale als auch Grenzen einer Übertragung zu erschließen. Dabei steht die Wechselseitigkeit möglicher Bürgerrollen von Konsumenten und Unternehmen im Mittelpunkt.

Schlagwörter: Bürgerschaft, Corporate Citizenship, Consumer Citizenship, politischer Konsum

\section{Political Responsibility in Market Arenas}

Transferring the idea of citizenship to actors of the market sphere implies an interconnectedness of political arenas and market arenas that are at tension with traditional concepts of citizenship. Applying the ideas of citizenship on corporations and consumers raises questions concerning the extent to which citizenship is a suitable category to conceptualise political action witbin the market sphere. Hence, the paper aims at scrutinising recent notions of consumer and corporate citizenship against the backdrop of the historical and philosophical principles of citizenship in order to reveal constraints and analytical potentials of using the term citizen with regard to market actors. Moreover, the contribution looks at the reciprocity of consumer citizenship and corporate citizenship.

Keywords: Citizenship, Corporate Citizenship, Consumer Citizenship, Political Consumerism

\section{Neukonfiguration des Bürgerbegriffs}

Mit der Übertragung auf die Konsumenten und Unternehmen erfährt der Bürgerbegriff eine Neukonfiguration, in der das grundlegende Spannungsverhältnis zwischen Politik und Ökonomie, Privatheit und Öffentlichkeit augenscheinlich wird. Entsprechend ambivalent stellen sich Einschätzungen einer politischen Rolle von Konsumenten und Unternehmen dar. Der Hervorhebung der Potenziale erweiterter Partizipationsmöglichkeiten, weicher Steuerungsformen und der Herausbildung transnationaler

Beitrag eingereicht am 21.06.2012; nach doppelt verdecktem Gutachterverfahren überarbeitete Fassung angenommen am 20.02.2013.

** Dr. Veronika Kneip, Frankfurt School of Finance \& Management, Sonnemannstr. 9-11, D-60314 Frankfurt a.M., Tel.: +49-(0)69-154008-365, Fax: +49-(0)69-154008-4365, E-Mail: v.kneip@fs.de, Forschungsschwerpunkte: Corporate Responsibility, Sustainability, Nongovernmental Organisations, Organisational Change. 
politischer (Markt-)Gemeinschaften stehen Befürchtungen einer Kommerzialisierung des Politischen und der Marginalisierung demokratisch legitimierter Herrschaft gegenüber. So verweist etwa Nikolas Rose auf neue Partizipationsformen durch die Gleichzeitigkeit von Konsumenten- und Bürgerrolle: „Citizenship is no longer primarily realized in a relation with the state, or in a single public sphere, but in a variety of private, corporate and quasi-public practices from working to shopping" (Rose 1999: 166). Benjamin Barber dagegen geht von einer vorgetäuschten Freiheit durch die „Privatisierung der Bürger" (Barber 2007: 118) in einer Verschmelzung mit der Konsumentenrolle aus. Nur der Staatsbürger übe legitime kollektive Gewalt aus und genieße eine tatsächliche, öffentliche Freiheit (Barber 2007: 167). Auch Colin Crouch hält fest „The consumer has triumphed over the citizen“ (Crouch 2004: 49), um damit ein wesentliches Charakteristikum der Postdemokratie und den für ihn damit verbundenen Verlust aktiver politischer Partizipation zu beschreiben. Mit Blick auf Unternehmen verhindert für Robert Reich die „Zurschaustellung unternehmerischer Tugendhaftigkeit" (Reich 2008: 252) dauerhafte Lösungen und verdeckt Probleme, mit denen sich eine Demokratie beschäftigen sollte. Dagegen stellt Dirk Messner die These auf, dass ,,[u]m die Globalisierung gestalten zu können, [...] auch die Problemlösungspotentiale privater Akteure, der Wirtschaft, der Gewerkschaften, von NGO mobilisiert werden" müssen (Messner 1998: 19).

In diesen unterschiedlichen Einschätzungen einer Gleichzeitigkeit von Konsumentenbzw. Unternehmens- und Bürgerrolle deuten sich erste Spannungsfelder an, die mit den Konzepten Consumer Citizenship und Corporate Citizenship verbunden sind. Diese Spannungsfelder werden im Rahmen dieses Beitrags genauer untersucht und systematisiert. Ausgehend von einem Überblick über beide Konzepte werden wesentliche Dimensionen von Bürgerschaft mit Blick auf eine mögliche Bürgerrolle von Konsumenten und Unternehmen untersucht. Zur Systematisierung wird auf die diametralen Rollenskripte zurückgegriffen, die mit der begrifflichen Trennung zwischen Citoyen und Bourgeois verbunden sind. Darauf aufbauend können sowohl Grenzen als auch analytische Potenziale der Übertragung des Bürgerbegriffs auf Konsumenten und Unternehmen beleuchtet werden. Ebenso kann aufgezeigt werden, inwieweit mögliche Bürgerrollen von Konsumenten und Unternehmen aufeinander bezogen sind und welche konkreten empirischen Fragestellungen sich daraus ergeben.

\section{Consumer Citizenship und Corporate Citizenship}

Hintergrund des Konzepts Consumer Citizenship ist die Berücksichtigung des politischen Kontextes materieller Güter und unternehmerischer Entscheidungen, die Michele Micheletti (2003: x) als „politics of products“ bezeichnet. Die Politik hinter den Produkten umfasst dabei verschiedene Facetten, die insbesondere im Zusammenhang mit dem Machtgewinn von Unternehmen und komplexen globalen Produktionsmechanismen stehen. Historisch ist die Verknüpfung von Bürger- und Konsumentenrolle zwar nicht an Globalisierungsprozesse gebunden - Boykotte im Zuge der AntiSklaverei-Bewegung in den USA Ende des 18. Jahrhunderts oder die britischen cooperatives der Arbeiterbewegung Anfang des 20. Jahrhunderts sind nur zwei Beispiele früherer Formen einer Politisierung des Konsums - allerdings erfolgt die Betrachtung von Konsumenten in einer Bürgerrolle vor allem vor dem Hintergrund der politischen Macht von Unternehmen im Globalisierungskontext: 
„[...] [C]onsumer critique is fundamental to citizenship in the age of globalization. It brings into the daylight the dangerously hidden issue of political power of corporations. [...] it exposes the potential power of consumers as citizens and provides incentives to businesses, which regulation increasingly does not, to mind corporate responsibility to and dependence on democracy." (Scammell 2000: 354)

Consumer Citizenship geht somit von einer Verschmelzung der Konsumenten- und Bürgerrolle im Sinne einer Übertragung bürgerschaftlicher Verhaltensweisen und Überlegungen auf Konsumhandlungen aus. Politisch motivierte Konsumhandlungen werden als Form demokratischer Partizipation verstanden, die dazu beitragen kann, gesellschaftliche Veränderungsprozesse anzustoßen oder zu bestärken. Im politischen Konsum handelt der Konsument als Bürger: „(...) [C]ivil action turns the sphere of consumption into a political terrain“" (Zadek/Amalric 1998: 11).

Ebenso wie der Staatsbürger die Wahl zwischen verschiedenen Parteien oder Kandidaten hat, wählt der Konsumentenbürger zwischen Produkten und Produzenten mit dem Ziel, Einfluss auf die Unternehmenspolitik einzelner Firmen und Konzerne oder auf übergreifende institutionelle Regulierungen $\mathrm{zu}$ nehmen: „campaigns that urge investors and consumers to express their ethics are asking people to vote with their money“ (Linton 2003: 361). Das Aktionsspektrum des Consumer Citizen bleibt jedoch nicht auf den reinen Kaufakt beschränkt. So grenzen Dietlind Stolle und Michele Micheletti (2005: 41) die direkt mit dem Kaufakt verbundenen Formen politischen Konsumentenhandelns, Boykott und Buykott, ${ }^{1}$ von diskursivem politischem Verbraucherhandeln ab, worunter Aktionen verstanden werden, die das Verhalten von Unternehmen in politischen, ethischen und ökologischen Fragen thematisieren. Trotz ihrer prinzipiellen Unterscheidbarkeit in kaufbasierte und kommunikationsbasierte Formen finden sich in der Praxis häufig Mischformen politischen Konsums. Eine solche Mischform stellen Boykottandrohungen oder medial inszenierte Boykottaufrufe dar, welche das Druckmittel öffentlicher Skandalisierung mit dem Aspekt monetärer Sanktionierung verknüpfen.

Während der Begriff Consumer Citizen den Konsumenten als Bürger beschreibt, steht der Begriff Corporate Citizen für die Betrachtung von Unternehmen als Bürger. Verschiedene Definitionen von Corporate Citizenship setzen dabei an der Verantwortungsübernahme des Unternehmens zur Lösung übergreifender gesellschaftlicher Probleme an: „(...) [C]orporate citizenship is about business taking account of its impact on both society and the environment, and about their being committed to delivering on social and environmental goods“ (Palacios 2004: 393). „Wie ein guter Bürger" (Westebbe/Logan 1995: 17) soll sich das Unternehmen in das Gemeinwesen einbringen, in dem es tätig ist und dort sozial, kulturell und ökologisch verantwortlich handeln. Neben seiner Rolle als Rechtssubjekt und der damit verbundenen Notwendigkeit der Einhaltung geltender Gesetze komme dem Unternehmensbürger eine aktiv gestaltende Rolle des eigenen Umfeldes zu, in deren Rahmen er negative externe Effekte bewusst vermeide und stattdessen auf die Erzeugung positiver externer Effekte

1 Während Boykott den bewussten Nicht-Kauf bestimmter Produkte oder Marken bezeichnet, kann unter Buykott der bewusste Kauf bestimmter Produkte (z.B. regionale Lebensmittel) oder Marken (z.B. TransFair-Siegel) verstanden werden. 
hinarbeite (Wieland 2003: 17). Hintergrund dieser Konzeption ist - analog zum Konzept Consumer Citizenship - die Überzeugung, dass Unternehmen als treibender Kraft der ökonomischen Globalisierung auch entscheidende politische Macht zukommt, mit der gesellschaftspolitisches Gestaltungspotenzial und eine entsprechende Verantwortung verbunden sind (z.B. Backhaus-Maul/Schubert 2005: 78; Matten et al. 2003: 115f.; Moon et al. 2008: 51f.).

Neben den Aspekten der Verantwortungsübernahme und Problemlösungskompetenz ist der strategische Charakter des bürgerschaftlichen unternehmerischen Engagements Ansatzpunkt unterschiedlicher Auseinandersetzungen mit dem Konzept. Dementsprechend ist von einer „mehr oder weniger strategisch ausgerichteten Investition in das natürliche und soziale Umfeld des Unternehmens“ (Heuberger et al. 2004: 3) oder einem „unternehmensstrategisch ausgerichteten Verständnis von Corporate Citizenship“ (Backhaus-Maul 2005: 239) die Rede. Die Wahrnehmung gesellschaftlicher Verantwortung wird mit der Verfolgung unternehmerischer Interessen verbunden. Das Unternehmen nutze die eigenen Kompetenzen, um sozialen Wandel herbeizuführen und trage so ,den wechselseitigen Abhängigkeiten von Unternehmen und gesellschaftlichem Umfeld systematisch Rechnung“ (Habisch 2003: 44). Schließlich wird unternehmerisches bürgerschaftliches Engagement analog zu einem bürgerschaftlichen Engagement von Individuen konzipiert, das mit Schlagworten wie Ehrenamt oder Freiwilligenarbeit charakterisiert werden kann. So sprechen Holger Backhaus-Maul und Ingolf Schubert (2005: 79) explizit von einem freiwilligen, ehrenamtlichen Engagement des Corporate Citizen. ${ }^{2}$

\section{Wechselseitigkeit von Consumer Citizenship und Corporate Citizenship}

Die vorangegangenen Skizzierungen der Begriffsverwendung von Consumer Citizenship und Corporate Citizenship haben einen ersten Einblick in relevante Aspekte beider Konzepte gegeben. Im Folgenden soll genauer untersucht werden, inwieweit sich die Konzeptualisierung dieser Rollen gegenseitig bedingt, inwieweit Aneignungsprozesse einer Bürgerrolle aufeinander bezogen sind und wie Interaktionen von Konsumenten und Unternehmen in einer Bürgerrolle gestaltet sein können. Ausgangspunkt hierfür ist eine Systematisierung bürgerlicher Rechte und Pflichten sowie der damit verbundenen Formen und Möglichkeiten der Partizipation bzw. der Erwartungen an das Handeln des Bürgers innerhalb des politischen Gemeinwesens. Zur Systematisierung wird auf die im romanischen Sprachraum entstandene begriffliche Unterteilung in Citoyen und Bourgeois zurückgegriffen. Der Begriff Citoyen leitet sich vom lateinischen Civis ab, während sich der Begriff Bourgeois - wie auch die deutsche Bezeichnung Bürger (Burgher) - etymologisch auf das Wort Bourg (Burg) zurückführen lässt. Die im Französischen bereits im 11. bzw. 12. Jahrhundert vorgenommene sprachlichen Unterscheidung war zunächst nicht mit verschiedenen Rollendefinitionen verbunden. Unter dem Einfluss der neuzeitlichen Souveränitätslehre sowie der Etablierung kapitalistischer Wirtschaftsstrukturen erfolgte jedoch im 17. Jahrhundert eine inhaltliche Abgrenzung der primär an individuellen Interessen orientierten priva-

2 Inwieweit Corporate Citizenship als ehrenamtliches Engagement jenseits einer auf das Kerngeschäft bezogenen Corporate (Social) Responsibility verstanden werden kann, ist umstritten. Dieser Aspekt wird in Kapitel 3.1 genauer ausgeführt. 
ten (Wirtschafts-)Bürgerrolle des Bourgeois von der öffentlich-politischen Rolle des Citoyen als Staatsbürger (Riedel 1972: 678; Rieger 1998: 94f.). Der begriffliche Dualismus geht einher mit unterschiedlichen Konturierungen von Bürgerschaft, die vor allem in den historischen Begründungslinien des Republikanismus und Liberalismus zum Ausdruck kommen, wenn auch nicht mit ihnen gleichgesetzt werden können. So sind liberale Entwürfe durchaus auf den Bürger als Staatsbürger bezogen, verknüpfen jedoch innerhalb dieser Konstellation die Bürgerrolle vielfach mit ökonomisch ausgerichteten Handlungslogiken. Während republikanische Theorien ihre Wurzeln in der Antike haben, sind liberale Vorstellungen ebenso wie die begriffliche Zweiteilung der Bürgerrolle mit der Herausbildung des modernen Territorialstaates verbunden. Bürgerschaft konstituiert sich in der republikanischen Tradition vor allem über die Teilhabe an der politischen Gemeinschaft, wohingegen das Individuum im Mittelpunkt des historischen Liberalismus steht. Im englischen Sprachraum wird entsprechend auch zwischen „civic republicanism“ und „liberal individualism" unterschieden (z.B. Oldfield 1998: 188f; Young 1995: 178). Allerdings gibt es sowohl innerhalb der republikanischen als auch innerhalb der liberalen Tradition unterschiedliche Ausprägungen; zudem versuchen neuere Ansätze, Aspekte des Republikanismus und Liberalismus miteinander zu verknüpfen und damit auch Bürgerschaft neu zu fassen.

\begin{tabular}{|c|c|c|}
\hline & \multicolumn{2}{|c|}{$\begin{array}{l}\text { Inhalte bürgerlichen Handelns } \\
\text { Erwartungen/Rollenvorgaben }\end{array}$} \\
\hline & CITOYEN & BOURGEOIS \\
\hline $\begin{array}{l}\text { Rechte und } \\
\text { Pflichten }\end{array}$ & $\begin{array}{l}\text { - Bürgerrechte als Rechte der gesamten } \\
\text { Polis } \\
\text { Betonung der Bürgerpflichten als } \\
\text { Solidarität gegenüber dem bürgerlichen } \\
\text { Gemeinwesen }\end{array}$ & $\begin{array}{l}\text { - } \quad \text { Betonung individueller Bürgerrechte } \\
\text { Bindung von Bürgerpflichten an } \\
\text { vertragliche Regelungen }\end{array}$ \\
\hline $\begin{array}{l}\text { Regieren und } \\
\text { regiert werden }\end{array}$ & $\begin{array}{l}\text { - Autonomie des bürgerlichen } \\
\text { - } \quad \text { Staat als Garant eines gelingenden Lebens }\end{array}$ & $\begin{array}{l}\text { - Selbstbestimmtheit des Individuums } \\
\text { - Staat als Zweckbündnis }\end{array}$ \\
\hline $\begin{array}{l}\text { Freiheit und } \\
\text { Gleichheit }\end{array}$ & $\begin{array}{l}\text { - Freiheit durch Integration und Beteiligung } \\
\text { - Konzentration auf faktische Gleichheit }\end{array}$ & $\begin{array}{l}\text { - } \quad \text { Freiheit durch Unabhängigkeit } \\
\text { Konzentration auf formale } \\
\text { Gleichheit/Zugangsoffenheit }\end{array}$ \\
\hline $\begin{array}{l}\text { Bürgertugend } \\
\text { und } \\
\text { Gemeinwohl }\end{array}$ & $\begin{array}{l}\text { - Bürgertugend als moralische } \\
\text { Grundhaltung } \\
\text { - Gemeinwohl als überindividueller Wert }\end{array}$ & $\begin{array}{l}\text { - Bürgertugend als regelkonformes } \\
\text { Verhalten } \\
\text { - Gemeinwohl als Aggregation individueller } \\
\text { Interessen }\end{array}$ \\
\hline
\end{tabular}

\section{Tabelle 1: Dimensionen des Bürgerbegriffs (eigene Darstellung)}

Tabelle 1 zeigt Extrempositionen bzw. idealtypische Rollenbeschreibungen für verschiedene Dimensionen des Bürgerbegriffs auf. ${ }^{3}$ Diese Extrempositionen - zusammengefasst als Rollenskripte des Citoyen und Bourgeois - können als Endpunkte eines Kontinuums möglicher Bürgerrollen verstanden werden. Die Systematisierung 
wird im Anschluss genutzt, um bestehende Ansätze der Übertragung des Bürgerbegriffs auf Konsumenten und Unternehmen daran zu spiegeln.

In der Gegenüberstellung der diametralen Rollenskripte werden Komplexität und Mehrdimensionalität des Bürgerbegriffs deutlich. Bürgerschaft wird in verschiedenen Konzeptionen unterschiedlich, vielfach sogar widersprüchlich, konturiert. Mit der Verwendung des Bürgerbegriffs im Kontext von Konsumenten und Unternehmen erfolgt eine Verschränkung von Wirtschaftssphäre und politischer Arena, die gleichzeitig mit einer - nicht immer widerspruchsfreien - Verknüpfung der Rollenvorgaben von Citoyen und Bourgeois verbunden ist:

„[...] the political consumer is supposed to reunite the Dr Jekyll and Mr Hyde of modernity: the bourgeois and the citoyen." (Holzer 2006: 408)

„[...] damit sind die theoretischen Pole der Corporate Citizenship-Debatte bestimmt, die jeweils nur ein geteiltes Verständnis von Bürgerschaft vorlegen: Entweder wird der Bourgeois durch den Citoyen vereinnahmt oder vice versa. Der Großteil des anglo-amerikanischen Diskurses und die praktische Umsetzung verlaufen im Zwischenraum, sind allerdings theoretisch inkonsistent [...].“ (Meckling 2004: 40, H.i.O.)

Charakteristika, Unterschiede und Widersprüche der Verschmelzung verschiedener Bürgerrollen in den Figuren des Consumer Citizen und Corporate Citizen sollen im Folgenden entlang der Dimensionen bürgerschaftlichen Handelns in den Blick genommen werden. Dabei wird die bisherige Auseinandersetzung mit Bürgerrollen von Konsumenten und Unternehmen untersucht und erweitert, ebenso wie Bezüge zwischen den Bürgerrollen von Konsumenten und Unternehmen hergestellt werden. So werden Wechselwirkungen und Spannungsfelder hinsichtlich der politischen Verantwortungsübernahme von Konsumenten und Unternehmen identifiziert und für empirische Fragestellungen fruchtbar gemacht.

\subsection{Rechte und Pflichten: Zwischen Ordnungsrahmen und Selbstverpflichtung}

Betrachtet man zunächst die Pflichten eines Corporate Citizen im Sinne der Reichweite von Unternehmensverantwortung ist die Abgrenzung zwischen den Begriffen Corporate Citizenship und Corporate Social Responsibility (CSR) hervorzuheben. Während einige Autoren beide Termini synonym verwenden (z.B. Banerjee 2008; Reimer 2004), unterscheiden andere Autoren hinsichtlich der Reichweite der damit verbundenen Konzeptionen. Hier kann auf Positionen verwiesen werden, die Corporate Citizenship als freiwilliges soziales und gesellschaftliches Engagement begreifen, das über die eigentliche Geschäftstätigkeit des Unternehmens hinausgehe, während Corporate Social Responsibility alle Bereiche der Unternehmenstätigkeit umfasse und sich auf die sozialen und ökologischen Folgen des operationalen Geschäfts selbst beziehe (z.B. Biedermann 2008; Westebbe/Logan 1995; Backhaus-Maul/Braun 2007; BackhausMaul/Schubert 2005). Zwar wird in diesem Zusammenhang auch darauf hingewiesen, dass beide Begriffe das gesellschaftliche Engagement von Unternehmen aus sich ergänzenden Perspektiven thematisieren (Backhaus-Maul et al. 2008: 20; BackhausMaul/Braun 2007: 5), die damit verbundene Trennung zwischen einer Verantwortungsübernahme für die Binnenwelt des Unternehmens, die durch gesetzliche Regulie- 
rungen geprägt ist, und einer freiwilligen Verantwortungsübernahme für die Außenwelt des Unternehmens erscheint jedoch problematisch. Die Grenze zwischen Binnen- und Außenwelt des Unternehmens verläuft fließend, da z.B. übergreifende gesellschaftliche Probleme, deren Lösung wesentlicher Bestandteil des zuvor skizzierten engen Verständnisses von Corporate Citizenship ist, durchaus im Kontext mit der operationalen Tätigkeit des Unternehmens stehen können. Eine Rolle des Unternehmens als (Good) Corporate Citizen kann dementsprechend kaum von einer Verantwortungsübernahme für die sozialen oder ökologischen Konsequenzen der unmittelbaren Unternehmenstätigkeit getrennt werden. Schlüssiger erscheinen dementsprechend Konzeptionen, in denen der Verantwortungsbereich der Unternehmensbürgerrolle umfassender definiert wird (z.B. Birch 2001; Schrader 2003; Thompson 2005; Wieland 2003). Corporate Social Responsibility ist nach Auffassung dieser Autoren integraler Bestandteil von Corporate Citizenship, wobei sich Corporate Citizenship nicht in Corporate Social Responsibility erschöpft. In Anlehnung daran kann die Verantwortungsübernahme für das unternehmerische Kerngeschäft (Corporate Social Responsibility) als die zentrale Pflichtdimension der Unternehmensbürgerrolle verstanden werden, welche durch die Dimension der Bürgerrechte sowie durch weitere Engagementformen außerhalb des unternehmerischen Kerngeschäftes ergänzt wird. Entsprechende Bürgerpflichten beziehen sich somit auf die hergestellten Produkte bzw. den Produktionsprozess und die damit verbundenen sozialen und politischen Konsequenzen. Mit der Formulierung dieser Unternehmensbürgerpflichten gehen allerdings auch Vorstellungen von Unternehmensbürgerrechten einher, die zum einen das Recht auf wirtschaftliche Betätigung und zum anderen das Recht auf ordnungspolitische Mitgestaltung umfassen. Während Ersteres vor allem „das Bürgerrecht, eigene Interessen zu verfolgen“ (Seitz 2002: 61) im Sinne des Eigentumsschutzes und der Gewinnmaximierung umfasst, bezieht sich Letzteres auf Formen der institutionellen Beteiligung im Politik- und Gesetzgebungsprozess - sowohl was das Politikfeld Wirtschaft als auch was Politikfelder wie Bildung, Kultur oder Soziales betrifft: „Citizenship in its usual sense not only imposes responsibilities and obligations on corporations but also confers the right to influence policy decisions" (Rondinelli 2002: 392). Hierin zeigt sich eine Verknüpfung der Rollenvorgaben von Bourgeois und Citoyen, da sowohl Besitzrechten als auch Partizipationsrechten wesentlicher Stellenwert eingeräumt wird.

Ebenso wie für Unternehmen Verantwortungsbereiche entlang ihrer Produktionsbzw. Wertschöpfungsstrukturen verortet wurden, können für die Konsumentenseite Verantwortungsbereiche identifiziert werden, die im Kontext ihrer Konsumhandlung stehen. In der Auseinandersetzung mit der Figur des Consumer Citizen machen verschiedene Autoren auf eine mögliche Verantwortung des Konsumenten durch die gesellschaftliche Relevanz der einzelnen Kaufentscheidung aufmerksam, die nicht nur die Wahl eines bestimmten Produktes beinhalte, sondern auch an dessen strukturellen Hintergrund (z.B. Arbeitsbedingungen an den Produktionsstandorten, ökologische Konsequenzen des Produktionsprozesses oder der Produktverwendung) gebunden sei (Barnett et al. 2005: 24; Holzer 2006: 407f.; Micheletti 2003: 11-15; Spaargaren/Martens 2005: 46-48; Stolle/Micheletti 2005: 14). Roger Dickinson und Mary Carsky betonen, dass eine Berücksichtigung entsprechender Verantwortungsbeziehungen auf der Loyalität innerhalb eines (transnationalen) politischen Gemeinwesens 
basiere und schließen somit an das Rollenskript des Citoyen an, um Konsumentenpflichten zu beschreiben. Gleichzeitig sprechen sie jedoch das Problem der Zurechenbarkeit einzelner Konsumhandlungen an:

„[...] [O]ur purchases can impact society. We may be promoting sweatshops in developing countries by our votes. And why not demand of consumers that they consider the ramifications of their actions on the common good, if that impact can be reasonably and readily ascertained?" (Dickinson/Carsky 2005: 31)

Darin wird deutlich, dass eine Definition der Rechte und Pflichten des Consumer Citizen immer des entsprechenden Bezugsrahmens bedarf, der, so die Autoren, auf der Grundlage von Informationen über Unternehmen und Produktionsprozesse rekonstruiert werden muss. Der Zugang zu Informationen stellt vor diesem Hintergrund ein zentrales Recht von Konsumenten in einer Bürgerrolle dar, das sowohl von politisch-institutionellen Akteuren als auch von Unternehmen eingefordert werden kann. Ebenso können Rechte auf Produktsicherheit oder auf den Schutz vor gesundheitsgefährdenden Produkten direkt mit Blick auf Unternehmen formuliert werden. Allgemeine Gültigkeit erhalten sie jedoch nur durch gesetzliche Regulierungen. Deren Bedeutung wird von einigen Autoren für Rechte wie den Schutz vor irreführender Werbung oder Monopolbildung bzw. für übergreifende Rechte wie ein ökologisch intaktes Umfeld oder die Repräsentation konsumrelevanter Themen im institutionellen politischen Prozess betont (Cohen 2003: 345f.; Gabriel/Lang 2006: 30f.; Spaargaren/Martens 2005: 31f.). Wie bereits für die Unternehmensseite zeigt sich mit Blick auf die Dimension der Bürgerrechte somit ein Rekurs auf die Rollen von Bourgeois und Citoyen, da für den Consumer Citizen sowohl individuelle Schutzrechte als auch umfassende Polisrechte formuliert werden.

Auch in der Zusammenschau der thematisierten Aspekte wird deutlich, dass bestehende Konzeptionen von Consumer Citizenship und Corporate Citizenship durchaus über Beschreibungen einer Marktbürgerrolle im Sinne des Rollenskripts des Bourgeois hinausgehen. Der Dimension von Bürgerpflichten wird ein wesentlicher Stellenwert eingeräumt und insbesondere durch die zentrale Bedeutung des Verantwortungsbegriffs wird an das Rollenmodell des Citoyen angeschlossen, für den eine proaktive Verantwortungsübernahme innerhalb des politischen Gemeinwesens handlungsleitend ist. Problematisch erscheint jedoch die teilweise fehlende Anbindung an Kontrollinstanzen bzw. an einen externen Bezugspunkt der jeweiligen Rechte und insbesondere Pflichten. Eine solche Anbindung ist mit Blick auf die Durchsetzbarkeit eines Kanons von Rechten und Pflichten wesentlich, wobei vor allem auf transnationaler Ebene nur bedingt Ordnungsrahmen existieren, in die eine Bürgerrolle von Konsumenten und Unternehmen eingebettet werden kann. Vor diesem Hintergrund kann die begrenzte institutionelle Einklagbarkeit von Bürgerrechten bzw. Kontrolle der Einhaltung von Bürgerpflichten als eine wesentliche Spannungslinie in der Übertragung des Bürgerbegriffs auf Konsumenten und Unternehmen identifiziert werden. Daraus resultiert die Notwendigkeit, das Verhältnis zwischen Selbstverpflichtungen bzw. selbst auferlegter Verantwortung und der Etablierung verbindlicher Ordnungsrahmen genauer zu untersuchen. Dabei sind nicht nur gesetzliche Vorgaben, sondern auch Prozesse der gesellschaftlichen Selbststeuerung zu berücksichtigen. Spezifische Analysen zur Wechselseitigkeit einer politischen Rolle von Konsumenten und Unternehmen 
können dabei bereits vorliegende Ergebnisse zu Governance-Systemen und Prozessen jenseits gesetzlicher Vorgaben ergänzen. Inwieweit kann etwa eine wechselseitige Bezugnahme von Konsumenten und Unternehmen in ihrer jeweiligen Formulierung von Bürgerrechten und -pflichten eine Kontrollfunktion übernehmen bzw. einen Kanon von Rechten und Pflichten etablieren? Relevant sind hier vor allem Prozesse der Zuschreibung von Verantwortung für bestimmte Produkteigenschaften und Produktionsverfahren sowie damit verbundene Fragen nach Produktkennzeichnungen oder unternehmerischer Berichterstattung.

\subsection{Regieren und regiert werden: Unternehmerische Machtausübung unter kollektiver Konsumentenkontrolle}

Eine Rolle von Konsumenten und Unternehmen als Regierte ist zunächst bestimmt durch den Einfluss nationaler bzw. transnationaler Ordnungsrahmen und ist für Konsumenten zudem eng mit ihrer Rolle als Staatsbürger verbunden. Mit den zuvor erläuterten Defiziten der verbindlichen Festschreibung von Rechten und Pflichten ist gleichzeitig eine Beschränkung der Rolle von Konsumenten und Unternehmen als Regierte verbunden. Zudem zeigen sich sowohl für Konsumenten als auch für Unternehmen Grenzen der Übertragbarkeit des Bürgerbegriffs hinsichtlich einer Rolle als Regierende im Sinne einer institutionalisierten Teilhabe in Form eines aktiven oder passiven Wahlrechts. Zwar können Unternehmen durch ihre Standortentscheidungen und Lobbyingbemühungen durchaus Einfluss auf die politische Willensbildung und Rahmensetzung nehmen, der direkte Einfluss auf die Herrschaftssicherung durch Wahl steht ihnen allerdings nicht zu. Auch für Konsumenten bleibt die Teilnahme an Wahlen ihrer Rolle als Staatsbürger vorbehalten. Allerdings können sie ebenfalls durch Lobbying und Gremienarbeit oder aber - in einer direkten Verknüpfung einer Staatsund Konsumentenbürgerrolle - durch die Wahrnehmung gesetzlich verankerter Klagerechte Einfluss auf staatliche Machtausübung nehmen.

Die Verwendung des Bürgerbegriffs für Marktakteure ermöglicht jedoch nicht nur die Betrachtung ihrer Rolle in Prozessen institutionalisierter Machtausübung. Vielmehr können Machtstrukturen in den Blick genommen werden, die nicht unmittelbar auf (Wahl-)Verfahren basieren. Jörn Lamla (2005: 7) verweist beispielsweise auf den Anspruch des Consumer Citizen, an der Regulierung gesellschaftlicher Prozesse mitzuwirken. Auch Ingo Schoenheit (2007: 216) grenzt den Begriff des politischen Konsums als Handlungsprogramm des Consumer Citizen von Begriffen wie qualitativem oder nachhaltigem Konsum durch dessen Zielsetzung ab, bestehende Zustände bzw. das Verhalten ,Anderer' zu beeinflussen und gesellschaftliche Veränderungen herbeizuführen. Entsprechend werden das Engagement für formelle Rahmenordnungen auf nationaler und globaler Ebene bzw. die Mitgestaltung gesellschaftlicher Verhältnisse als wesentliche Bestandteile einer Unternehmensbürgerrolle bezeichnet (z.B. Backhaus-Maul 2005: 239; Habisch/Schwarz 2012: 126; Seitz 2002: 107). Ebenso werden unter Corporate Citizenship „Praktiken der Steuerung und des Regierens“ (Sack 2005: 176) verstanden und Unternehmensbürger als „Mitgestalter der Weltpolitik“ (Behrent 2003: 24) bezeichnet. Für beide Akteursgruppen wird somit eine Rolle als ,Regierende definiert, wobei eine Rolle des Consumer Citizen als Regierender im Wesentlichen darauf zielt, die Machtposition von Unternehmen zu begrenzen bzw. zu lenken. 
In diesem Zusammenhang kommt der kollektiven Dimension einer politischen Rolle von Konsumenten große Bedeutung zu. Diese kollektive Dimension nimmt in bestehenden Entwürfen von Consumer Citizenship einen wesentlichen Stellenwert in der Auseinandersetzung mit Möglichkeiten der Machtausübung und -kontrolle außerhalb des institutionalisierten Politikprozesses ein:

„Yet the power behind political consumerism is not the power of the individual consumer, but the power of agencies that command enough credibility to influence many people's decisions and thus to transform individual choices into a collective statement [...].“ (Holzer 2006: 407)

Die Kollektivierung, so die These, trage entscheidend zu einer Politisierung des Konsums im Sinne einer gesellschaftlichen Gestaltungsfunktion bei. Terrence Guay et al. (2004: 132f.) heben beispielsweise mit Blick auf Shareholder-Aktivismus und ethisches Investment die Expertise- und Bündelungsfunktion von Nichtregierungsorganisationen hervor. Kollektive bzw. kollektivierte Konsumhandlungen werden insgesamt sowohl aufgrund ihrer Effektivität als auch aufgrund der Integration in einen gemeinsamen Werthorizont als steuerungswirksam und als Ausdruck einer Bürgerrolle betrachtet. Für Michael Beetz (2005: 3) etwa agiert der Consumer Citizen als ,generalisierter Anderer des Wirtschaftssystems“, dessen Potenzial zur Herbeiführung politischer Reformen nicht der Macht als privater Einzelner, sondern dem Kollektiv der Verbraucheröffentlichkeit entspringe. Diesen Machtaspekt der kollektivierten Verbraucherwahl betont auch Boris Holzer (2006: 405f.) als „,collective' of aggregated and communicated choice". Jörn Lamla (2005: 7) schließlich stellt die Regulierungskraft des politischen Konsums aus gesellschafts- und demokratietheoretischer Perspektive in Frage, sofern dieser auf eine individualisierte und in der Alltagsökonomie verankerte Bürgerkultur beschränkt bleibt. Damit basieren Entwürfe der Konsumentenbürgerschaft wesentlich auf der Idee der Einbeziehung Aller in Prozesse des Regierens, womit gleichzeitig eine Kontrollfunktion des Kollektivs im Sinne des RegiertWerdens verbunden ist.

In Konzeptionen der Unternehmensbürgerschaft wird sowohl auf kollektive als auch auf individuelle Dimensionen der Machtausübung und -kontrolle Bezug genommen. So können zunächst Positionen angeführt werden, die auf die Bedeutung kollektiver Aspekte verweisen, um Unternehmen in einer Bürgerrolle betrachten zu können. Die aktive Interaktion mit verschiedenen Akteuren bzw. Akteursgruppen der Unternehmensumwelt stellt etwa für Philip Mirvis und Bradley Googins (2009: 6f.) das zentrale Element einer „,next generation of corporate citizenship“ dar, die das Potenzial habe, sozialen Wandel herbeizuführen. Auch für André Habisch ist der ordnungspolitische Gestaltungsanspruch von Unternehmen nur in Kooperation mit anderen Akteuren umsetzbar; er verweist auf „Kooperationsprojekte zwischen Unternehmen und mindestens einem Partner aus einem anderen gesellschaftlichen Bereich, die auf die Lösung gesellschaftlicher Probleme bezogen sind“ (Habisch 2003: 53). Ebenso weist Simon Zadek darauf hin, dass (freiwillige) Maßnahmen einzelner Unternehmen nicht ausreichend seien, um strukturelle soziale oder ökologische Probleme zu lösen und plädiert für ein kollektives Vorgehen gemeinsam mit staatlichen und zivilgesellschaftlichen Akteuren in einer "Third Generation Corporate Citizenship“ (Zadek 2007, 2002). Generell umfasst die Auseinandersetzung mit kollektiven Formen der Macht- 
ausübung auch für den Corporate Citizen nicht nur den Aspekt des Regierens, sondern auch den des Regiert-Werdens. Prozesse kollektiver Selbststeuerung sind mit Aspekten kollektiver Selbstbindung verknüpft, da die Festlegung und Durchsetzung gemeinsamer Regeln die Anerkennung externer Kontrolle durch die an der Kooperation beteiligten Akteure beinhaltet. Eine Bereitschaft zur kollektiven Selbstbindung stellt vor allem Peter Ulrich in den Mittelpunkt seiner Auseinandersetzung mit der Bürgerrolle von Unternehmen. So fordert er vom Corporate Citizen:

„[...] [D]ie Bereitschaft, gemeinwohldienliche ordnungspolitische Reformen auch dann mitzutragen, wenn sie vom eigenen Unternehmen bzw. der eigenen Branche ein zumutbares Opfer (Kostenfolgen) verlangen, statt Politik nur lobbyistisch als die Fortsetzung des Geschäfts mit anderen Mitteln zu betreiben.“ (Ulrich 2000: 18)

Jem Bendell (2000: 249-251) verknüpft seinen Entwurf von „Radical Corporate Citizenship“ sogar explizit mit der Forderung nach externen Kontrollen im Rahmen von Global-Governance-Konstellationen, die Unternehmen in eine Rolle als ,Regierte (zurück-)versetzen:

„Radical corporate citizenship suggests that companies should begin by submitting to the authority of fledgling intergovernmental processes and by not lobbying against their regulatory functions. Moreover, there is good reason for companies to lobby for heightened regulation at the intergovernmental level in order to raise the common standard of practice and ensure greater benefit for all."

(Bendell 2000: 251)

Dagegen kann die Hervorhebung einer ,individuellen' Selbstbindung ${ }^{4}$ des einzelnen Unternehmens, wie sie in anderen Ansätzen zu finden ist, eher im Sinne der Rollenbeschreibung des Bourgeois als Rekurs auf Unabhängigkeit und Selbstbestimmung verstanden werden. So verweist Holger Backhaus-Maul (2004: 23, 28) auf den Anspruch von Unternehmen, ihre Engagementbereiche und -projekte unabhängig von externer Einflussnahme selbst zu wählen. Ähnlich stellt für Simone Paar (2005: 4f.) die angestrebte Mitgestaltungsmöglichkeit des Unternehmens vor allem einen Gewinn für dessen Eigenständigkeit dar, da Corporate Citizens entscheidenden Einfluss auf die Gestaltung des eigenen Handlungsspielraums nehmen könnten.

Die hier offensichtliche Spannungslinie zwischen kollektiver und ,individueller' Selbststeuerung prägt die Dynamik von Corporate Citizenship und Consumer Citizenship. Wesentlich ist dabei, die Machtverteilung zwischen den verschiedenen Akteuren bzw. Akteursgruppen einzubeziehen, da hier die Wechselseitigkeit möglicher Bürgerrollen von Konsumenten und Unternehmen deutlich wird. Diese Machtposition wird auch in einigen bisherigen Entwürfen von Consumer Citizenship und Corporate Citizenship diskutiert. Mit Blick auf Konzeptionen von Corporate Citizenship kann hier vor allem auf Arbeiten verwiesen werden, in denen von einer staatenähnlichen Machtposition von Unternehmen ausgegangen wird. Insbesondere Andrew Crane, Dirk Matten und Jeremy Moon haben eine entsprechende Interpretation von Corporate

4 „Individuell“ kann an dieser Stelle nur „bezogen auf das einzelne Unternehmen“ bedeuten. Die Frage, inwieweit Unternehmen als Nicht-Individuen bzw. Kollektivakteure prinzipiell als Bürger bezeichnet werden können, wird z.B. von Michaël Gonin (2006: 42-46) thematisiert. 
Citizenship vorangetrieben (Crane et al. 2008; Crane et al. 2004; Matten/Crane 2005; Matten et al. 2003; Moon et al. 2008). Für sie drückt dieser Begriff weniger die Position von Unternehmen als Bürger innerhalb eines politischen Gemeinwesens als vielmehr das Verhältnis von Unternehmen zum Konzept der Bürgerschaft in dem Sinne aus, dass Unternehmen im Zuge des Steuerungsverlustes von Nationalstaaten selbst zum Garanten der Bürgerrechte ihrer Anspruchsgruppen (Mitarbeiter, Konsumenten, etc.) geworden sind, welche Dirk Matten und Andrew Crane in Anlehnung an den liberalen Theoretiker Thomas Marshall in zivile, politische und soziale Rechte unterscheiden:
„Our premise is that corporations enter the arena of citizenship in circumstanc- es where traditional governmental actors fail to be the ,counterpart ${ }^{6}$ of citizen- ship. [...] corporations have tended to partly take over (or are expected to take over) certain functions with regard to the protection, facilitation, and enabling of citizens' rights-formerly an expectation placed solely on governments. “ (Mat- ten/Crane 2005: 171)

Eine solche Rolle als ,Regierende ${ }^{5}$ wird Unternehmen vor allem dort zugeschrieben, wo Staaten sich aus ihrer Verantwortung zurückziehen, wo sie die Verwirklichung von Bürgerrechten noch nicht übernommen haben oder wo sie prinzipiell nicht dazu in der Lage sind (Matten et al. 2003: 116f:; Moon et al. 2008: 60-62). Crane et al. (2008: 88-122; 2004: 110-114), Moon et al. (2008: 63-65) und Maak (1999: 269) konzeptualisieren außerdem Handlungen verschiedener Anspruchsgruppen des Unternehmens ausdrücklich als politische Partizipation in einer Bürgerrolle und weisen auf die Kontrollfunktion dieses Handelns hin. Damit gehören diese Autoren zu den wenigen, die eine Verknüpfung unterschiedlicher Marktbürgerrollen berücksichtigen.

Der in diesen Konzeptualisierungen anklingende Machtvorsprung von Unternehmen in den entlang von Produktionsketten strukturierten politischen Arenen, der sie in eine Rolle als ,Regierende' versetzt, ist auch Gegenstand der Auseinandersetzung mit einer möglichen Bürgerrolle von Konsumenten. Die dominante Position von Unternehmen ist dabei Ausgangspunkt von Überlegungen zur Notwendigkeit einer politisch aktiven Rolle von Konsumenten. So betrachtet Victoria Baxter (2003: 19) die Restrukturierung politischer Gestaltungsmacht zugunsten transnationaler Konzerne als treibende Kraft für die Verlagerung von Fragen des Gemeinwohls in die Konsumsphäre. Ebenso lässt die Konzeption des politischen Konsums als Wablakt auf Vorstellungen des Unternehmens als ,regierend“'schließen, da dieses in der Regel als Adressat einer Wahl mit dem Einkaufswagen gilt: „Consumers use their purchasing power as a kind of vote and thus are capable of successfully scandalising corporate giants like Shell, Nike or Monsanto in collective action“" (Baringhorst 2008: 175). In der Beschäftigung mit Consumer Citizenship steht jedoch nicht nur die direkte Interaktion zwischen Unternehmen und Konsumenten(-kollektiven) im Mittelpunkt; vielmehr wird auch die Bedeutung politischer Institutionen angesprochen, die politische (Kontroll-)Funktion von Konsumenten zu stärken und Unternehmen in ihrer Rolle als ,Regierende` direkt

5 In ihren Beiträgen aus dem Jahr 2008 sprechen Crane et al. bzw. Moon et al. explizit von „Corporations as Governments“ (Crane et al. 2008: 50; Moon et al. 2008: 60), während diese Rolle zuvor mit „Corporations as administrators of citizenship“ (Crane et al. 2004: 109) bzw. „Social role in administering citizenship rights“ (Matten/Crane 2005: 174) umschrieben worden war.

zfwu 14/1 (2013), 62-85 
einzuschränken (Bakan 2005; Baxter 2003; Littler 2009: 68f.; Oliviero/Simmons 2002). Mads Sorensen (2005: 65-67) macht in diesem Zusammenhang auf die Gefahr aufmerksam, dass der gesetzliche Ordnungsrahmen und damit verbundene Mechanismen der Politikimplementation angesichts von Selbstregulierungsprozessen durch Marktakteure ihre Bedeutung verlieren können und zeigt damit eine wesentliche Grenze der Bürgerrolle von Marktakteuren auf. Machtausübung und -kontrolle in transnationalen marktförmig strukturierten politischen Arenen muss als Zusammenspiel und wechselseitige Herausforderung unterschiedlicher (Markt-)Akteure in den Blick genommen werden. Dabei gilt es sowohl zu untersuchen in welcher Weise Unternehmen eine (externe) Legitimation ihrer Rolle als ,Regierende in den entlang von Produktionsprozessen strukturierten politischen Arenen anstreben als auch inwieweit Unternehmen durch Konsumenten(-kollektive) bzw. (trans-)nationale Ordnungsrahmen an ihre Rolle als Regierte gebunden werden können. Inwieweit öffnen oder verschließen sich Unternehmen beispielsweise gegenüber einem externen Monitoring ihrer transnationalen Produktionsprozesse? Welche Rolle spielen Konsumenten und die sie vertretenden Nichtregierungsorganisationen dabei, Transparenz und Rechenschaft einzufordern?

\subsection{Freiheit und Gleichheit: Ökonomische Ungleichheit und divergierendes Freiheitsverständnis}

Für Konsumenten und Unternehmen ergeben sich Übertragungsprobleme des Bürgerbegriffs sowohl mit Blick auf die Forderung nach formaler als auch nach faktischer Gleichheit. Der Anspruch, durch den Bürgerstatus die Mitgliedschaft in einer Gemeinschaft von formal Gleichen zu regeln, kann sowohl innerhalb der jeweiligen Akteursgruppe als auch mit Blick auf politische Marktarenen, in denen Konsumenten und Unternehmen auftreten, nur bedingt erfüllt werden. Zwar ermöglicht gerade die Ablösung des Bürgerstatus von staatlicher Zuschreibung eine breite Integration und wird in der Thematisierung der Bürgerschaft von Konsumenten teilweise sogar als Indiz für eine höhere Zugangsoffenheit gewertet: „The politicization of products means that a greater number of actors from different spheres are seen as playing a public responsibility-taking role in the world today" (Micheletti 2003: 13). Dennoch bleibt die formale Gleichheit an Institutionen gebunden, durch die eine solche Gleichheit festgeschrieben und umgesetzt wird. Für den Unternehmensbürger ergibt sich zudem das Problem komplexer und unterschiedlicher Unternehmenskonstellationen, die einer formalen Gleichheit verschiedener Unternehmen widersprechen.

Was den Aspekt faktischer Gleichheit also die tatsächlichen Handlungsmöglichkeiten betrifft, erscheint das Rollenskript des Citoyen sowohl für Konsumenten als auch für Unternehmen aufgrund der unterschiedlichen ökonomischen Ressourcenausstattung der jeweiligen Akteure problematisch. Dieser Aspekt wird in der Forschungsliteratur zu Corporate Citizenship nur teilweise bzw. untergeordnet thematisiert. Eine Ausnahme stellt beispielsweise der Ansatz von Adrian Schawalder (2006: 18) dar, der auf Einschränkungen (wirtschafts-)bürgerlicher Grundrechte durch die ungleiche ökonomische Position der Wirtschaftssubjekte hinweist. Dirk Matten et al. (2003: 117f.) problematisieren außerdem die ungleiche Machtverteilung zwischen Unternehmen und anderen Akteuren der jeweiligen politischen Marktarena. Mit Blick auf die Mög- 
lichkeit von Unternehmen, aufgrund ihrer ökonomischen Ressourcen Einfluss auf politische Strukturen zu nehmen, spricht schließlich Ronnie Lipschutz Aspekte der Ungleichheit an und weist auf die damit verbundene Gefahr einer Entdemokratisierung politischer Entscheidungen hin: „If capital is able to acquire political power, it is more a form of displacement than an alternative: the ,corporate citizen' becomes, in a sense, a franchisee able to cast a vote using its dollars" (Lipschutz 2003: 34).

Deutlicher wird die Problematik unterschiedlicher Ressourcenausstattung in Konzeptionen von Consumer Citizenship thematisiert. Faktische Ungleichheit wird hier im unterschiedlichen Potenzial, der eigenen politischen Stimme im Konsum Ausdruck zu verleihen, deutlich. Insbesondere bei Hochpreis- und Luxusgütern oder auch mit Blick auf Aktionäre als Consumer Citizens, so z.B. Neil Carter und Meg Huby (2005: 257), bleibt die direkte Stimmabgabe einer konsumkräftigen Minderheit vorbehalten. Aus dem damit verbundenen ungleichen Handlungsspielraum werden allerdings unterschiedliche Konsequenzen abgeleitet. Mads Sorensen (2005: 63) beispielsweise stellt Vorstellungen einer Verbraucherdemokratie aufgrund der ökonomischen Ungleichheit der Stimmenden in Frage:

„In this perspective, political consumerism appears profoundly undemocratic: in the market, where the political consumer acts, the votes are of course not distributed according to the principle of one vote each. On the contrary, each person has as many votes as his or her private economy allows." (Sorensen 2005: 63)

Roger Dickinson und Mary Carsky dagegen schließen aus einer ungleichen Ressourcenausstattung auf eine höhere Verantwortung derjenigen Konsumentenbürger, die über eine höhere ökonomische Stimmkraft verfügen: „We argue that consumers, particularly rich consumers, should do more than maximize their utilities" (Dickinson/Carsky 2005: 26). Auch empirisch kann untersucht werden, inwieweit ökonomische Ungleichheit Handlungschancen strukturiert und wie diese Ungleichheit von unterschiedlichen Akteuren zur Diskussion gestellt wird. Bereits vorliegende Analysen zum Thema Lobbying (z.B. Lee/Baik 2010; Svendsen 2011) können hier einen wichtigen Ansatzpunkt liefern, da sie ungleiche ökonomische Ressourcenausstattung verschiedener Akteure thematisieren und kritisieren. Ebenso ist der Aspekt der Ungleichheit relevant für die Frage nach der Etablierung von Bio- oder Fair-Trade-Produkten. Strukturelle Veränderungen in Produktionsprozessen sind hier nicht zuletzt an die ökonomischen Ressourcen von Konsumenten gebunden.

In diesem Sinne ist in der konzeptionellen Auseinandersetzung mit einer Bürgerrolle von Konsumenten etwa für Yiannis Gabriel und Tim Lang die der ökonomischen Konsumentenwahl zugrunde liegende Ungleichheit unmittelbar mit einem Freiheitsverlust verbunden: „So there is choice, but not equality of choice [...]. The key barrier to consumer choice is money. [...] Money gives choice. Choice gives freedom" (Gabriel/Lang 2006: 30). Auch Roger Dickinson reflektiert ökonomische Ungleichheit vor dem Hintergrund der Dimension der Freiheit, wobei er die Ungleichheit zwischen Unternehmen und Konsumenten in den Mittelpunkt stellt. Diese Ungleichheit, so Dickinson, könne vor allem durch die Integration von Konsumenten in Kollektive und den damit verbundenen Gewinn an Handlungsfreiheit abgeschwächt werden (Dickinson 1996: 262). Damit betont Dickinson den Aspekt der Freiheit, die auf der Integration in ein politisches Gemeinwesen und dem damit verbundenen Gestaltungs- 
spielraum des Kollektivs basiert. Allerdings wird in Konzeptionen einer Konsumentenbürgerrolle Freiheit durchaus auch durch individuelle Unabhängigkeit verwirklicht gesehen. So betont etwa Michele Micheletti einen Freiheitsgewinn des Consumer Citizen gegenüber dem Staatsbürger durch dessen unvermittelte und erweiterte Handlungsmöglichkeiten bzw. eine entsprechende Handlungsautonomie: „Involvement usually comes in a DIY (do-it-yourself) fashion: people want to decide for themselves what matters they want to work with and how they want to do so" (Micheletti 2004: 23). Auch in Entwürfen der Unternehmensbürgerschaft wird Freiheit sowohl mit Blick auf Integration als auch mit Blick auf Unabhängigkeit thematisiert. Hinsichtlich eines Freiheitsverständnisses, das auf einem kollektiv gewonnenen Handlungsspielraum basiert, können beispielhaft die Ausführungen von André Habisch (2003: 6165), Adrian Schawalder (2006: 17) und Peter Ulrich (2000: 7) genannt werden, die aus kollektiven Selbstbindungen einen ebenso kollektiven Freiheitsgewinn ableiten:

„Wirtschaftsbürgerethik lässt sich als Selbstbindung interpretieren und zielt darauf ab, den nicht erwünschten Auswirkungen ökonomischen Handelns entgegenzuwirken und Effizienz nur soweit zuzulassen, als sie von allen möglicherweise betroffenen Wirtschaftsbürgern als legitim anerkannt wird. Diese Selbstbindung wird nicht als unzumutbare Beschränkung aufgefasst, sondern als Grundvoraussetzung der grösstmöglichen Freiheit aller.“ (Schawalder 2006: 17)

Demgegenüber stehen Positionen, welche die Freiwilligkeit einer Unternehmensbürgerrolle in den Mittelpunkt stellen und dementsprechend Freiheit als ,individuelle Handlungs- und Entscheidungsfreiheit unter Abwesenheit externer Zwänge konzipieren:

„Gesellschaftspolitisch betrachtet handelt es sich beim Corporate Citizenship um die freiwillige Übernahme gesellschaftlicher Verantwortung durch private Unternehmen vor dem Hintergrund wirtschaftspolitisch günstiger Rahmenbedingungen." (Backhaus-Maul 2004: 24)

Corporate Citizenship wird dabei mit einem weiten Spielraum verbunden, was die zeitliche, sachliche und soziale Schwerpunktsetzung der Bürgerrolle betrifft (z.B. Polterauer 2005: 107; Sack 2005: 175). Die Betonung von Freiheit verstanden als Freiwilligkeit bzw. Unabhängigkeit muss allerdings vor dem Hintergrund des Anspruchs auf ordnungspolitische Mitgestaltung verstanden werden. Insbesondere angesichts der zuvor problematisierten Machtposition von Unternehmen kann sich die Wahrnehmung eines Freiheitsrechts durch den Unternehmensbürger als Ablehnung von Kontrolle darstellen. Aber auch für die Konsumentenseite stellt sich die Frage, wie Rollenvorgaben von Citoyen und Bourgeois miteinander verknüpft werden können. Inwieweit, so eine empirische Fragestellung, kann individuelle Wahlfreiheit mit (transnationaler) Solidarität einhergehen? Welche negativen externen Effekte mit Blick auf Arbeitsbedingungen und Ökologie sind etwa mit der Freiheit des Konsumenten verbunden, über immer kürzer werdende Produktlebenszyklen Bedürfnisse unmittelbar zu befriedigen? 


\subsection{Bürgertugend und Gemeinwohl: Gemischte Motive und Streben nach gesellschaftlicher Anerkennung}

Hinsichtlich der Dimension „Bürgertugend und Gemeinwohl“ stellen sich die zuvor aufgeworfenen Fragen noch einmal drängender. Besonders deutlich kann hier in den bestehenden Konzeptionen eine Verschränkung unterschiedlicher Rollenvorgaben identifiziert werden. So ist für Michele Micheletti die Rolle des Consumer Citizen mit der Konzentration auf öffentliche Tugendideale (public virtue tradition of politics) verbunden, worunter sie die Anbindung an gemeinwohlorientierte Interessen und eine tiefergehende politische Motivation versteht (Micheletti 2003: 19f.). Demgegenüber stellt sie private Tugendideale (private virtue tradition of politics) als weitere mögliche Antriebskraft von Konsumentenhandeln, das ebenfalls politisch wirksam werden kann. Hier bringt der Konsument das private Interesse in die Konsumhandlung ein, um Unternehmenspolitik bzw. politisch-institutionelle Strukturen zu verändern, so dass letztlich auch dieses - zunächst privat motivierte - Handeln Ausgangspunkt gesellschaftlichen Wandels sein kann (Micheletti 2003: 20f.). Auch Kate Soper geht von einer möglichen Verknüpfung unterschiedlicher Bürgerrollen im politischen Alltagshandeln aus: „A primarily egoistic rationale for changing consumption will very often be coloured by something more altruistic, and vice versa“ (Soper 2008: 195). Jacob Rosenkrands (2004: 59) spricht in diesem Zusammenhang von einem neuen Idealtyp als Mischung zwischen „Homo economicus" und „Homo politicus.

Unabhängig von der Anerkennung gemischter Motive sind mit Entwürfen von Consumer Citizenship in der Regel Vorstellungen eines überindividuellen Gemeinwohls verbunden. Demgemäß muss für Roger Dickinson eine Bürgerrolle von Konsumenten mit der Orientierung an übergeordneten Werten einhergehen: „In consumer votes the common good is to be recognized" (Dickinson 1996: 263). Diese überindividuelle Orientierung soll vor allem mit einer solidarischen, advokatorischen Vertretung benachteiligter bzw. schwächerer Gruppen einhergehen, die ihre Position nicht selbst über eine ökonomische Wahl oder öffentlichen Druck durchsetzen können (z.B. Clarke et al. 2007: 242; Littler 2009: 23-49). Michele Micheletti und Dietlind Stolle (2007: 166f.) verweisen mit Blick auf eine advokatorische Interessenvertretung konkret auf die Möglichkeit, als Consumer Citizen zur Stärkung der Position von Gewerkschaften gegenüber Unternehmen beizutragen. Eine Analyse entsprechender (Kooperations-)Beziehungen erscheint als relevanter Gegenstand für empirische Studien, um mögliche Spannungen zwischen einer advokatorischen und einer eigenwohlorientierten Konsumentenrolle bzw. universalen und partikularen Interessen zu identifizieren und hinsichtlich ihrer Konsequenzen für die Steuerbarkeit politischer Marktarenen zu hinterfragen.

Auch die Auseinandersetzung mit der Figur des Corporate Citizen greift sowohl eigenwohlorientierte als auch gemeinwohlorientierte Motive bürgerschaftlichen Engagements auf. Prominenten Stellenwert nimmt hier zunächst eine Argumentation ein, die den Unternehmensbürger in einer Rolle als Bourgeois sieht und die Leitidee unternehmerischen Gewinnstrebens als legitimes Bürgerrecht deutet:

„Corporate Citizenship bezeichnet das aktive Streben nach umfassenderer Nutzung des sozialen und natürlichen Umfelds, ausgehend von der Einsicht, daß Gewinnmaximierung Investitionen und also die Besserstellung der Interaktions- 
partner regelmäßig voraussetzt. Corporate Citizenship heißt, nach Chancen der Investition in die Gesellschaft aktiv und mit angemessener Methodik zu suchen." (Seitz 2002: 64)

Verbunden ist dieses Gewinnstreben mit einer punktuellen und freiwilligen Integration in das Gemeinwesen auf Grundlage eines individuellen Kosten-Nutzen-Vergleichs. Bürgerliches Engagement wird als Investition betrachtet, von der ein angemessener Ertrag zu erwarten ist: „So geht der Corporate Citizen oder korporative Bürger in Vorleistung und investiert gemeinsam mit anderen Akteuren in das Gemeinwesen" (Paar 2005: 3, H.i.O.). In diesem Zusammenhang werden in der Literatur verschiedene Gründe wie die Gewinnung relevanter Marktinformationen, die Stärkung der unternehmerischen Kompetenzen und Unternehmenskultur, erhöhtes Kundenvertrauen, höhere Mitarbeiterbindung oder die Herstellung externer Kontakte genannt, die den eigenwohlorientierten Corporate Citizen zu einer solchen Investition bewegen können (Lin 2010; Habisch 2003: 74-77; Janes/Stuchtey 2008: 24f.; Marsden/Andriof 1998: 340f.; McIntosh et al. 1998: xxi; Polterauer 2005: 106f.; Willmott 2003: 366). Peter Ulrich dagegen fordert von Unternehmen in einer Bürgerrolle eine innere moralische Grundhaltung, die er an Vorstellungen eines überindividuellen Gemeinwohls knüpft:

„Geboten ist somit eine Selbstbegrenzung des unternehmerischen Gewinnstrebens nach Massgabe ,unantastbarer' moralischer Rechte von Betroffenen und anderer höherrangiger Güter des republikanischen Gemeinwohls: Legitimes Gewinnstreben ist stets moralisch begrenztes Gewinnstreben.“ (Ulrich 2000: 16)

Eine wichtige Mobilisierungskraft zur Vertretung gemeinwohlorientierter Interessen liegt dabei nach Meinung Ulrichs im Streben nach Erhaltung der Selbstachtung bzw. im Bedürfnis, auch für andere ein achtenswerter Bürger zu sein, begründet (Ulrich 2005: 31). In ähnlicher Weise weist Stefan Nährlich eine Fokussierung auf Corporate Citizenship als „business case“ zurück. Vielmehr müsse ein angestrebter business case im Gleichgewicht mit einem „social case“ stehen, der sich nicht zwangsläufig aus jedweder Form des Engagements ergebe: „Jede Art von Engagement für die Gesellschaft erfährt ihre Legitimation, Akzeptanz und Anerkennung durch den gemeinwohlförderlichen Beitrag, der mit dem Engagement geleistet werden soll, nicht aufgrund der Tatsache, dass der Engagierte (auch) etwas davon hat" (Nährlich 2008: 27). Auch weitere normative Anforderungen an den Unternehmensbürger rekurrieren auf die Notwendigkeit gesellschaftlicher Anerkennung. So verknüpfen etwa Philipp Schwertmann (2005: 203) oder Chris Marsden und Jörg Andriof (1998: 337) die Motive bürgerlichen Engagements mit dem Streben nach Selbstlegitimation. Demgegenüber können Positionen angeführt werden, die gerade eine strategische Ausrichtung der Unternehmensbürgerrolle als gemeinwohlförderlich betrachten. Eine strategische Ausrichtung der Bürgerrolle verhindere, dass Engagement zum reinen „Luxusgut“ (Habisch 2003: 60) werde und diene einem übergeordneten Gemeinwohl, da aus einer strategischen Herangehensweise eine langfristige und verbindlichere Ausrichtung resultiere. In diesem Sinne zeigt sich für Grahame Thompson (2005: 135) Verantwortungsübernahme für das Gemeinwesen in einem Engagement, das über punktuelle Philanthropie hinausgeht und im Kern der Geschäftsstrategie verankert ist. Auch Malcolm McIntosh et al. betonen den Aspekt der Nachhaltigkeit, der mit einer strategischen Ausrichtung der Bürgerrolle verbunden sei: ,'The new corporate citizenship is 
not an afterthought managed by public relations. The new corporate citizenship is about citizenship at the heart of strategic planning“" (McIntosh et al. 1998: 35).

Strategisches Kalkül des Corporate Citizen erscheint somit als Voraussetzung, um sowohl den Unternehmensbürger bei der Ressourcengewinnung zu unterstützen als auch dem politischen Gemeinwesen eine effektive Versorgung mit öffentlichen Gütern zu ermöglichen. Die so vorgenommene Verknüpfung der Rollenbeschreibungen von Citoyen und Bourgeois wirft jedoch konzeptionelle Probleme auf und wird von Jonas Meckling zu Recht als theoretisch inkonsistent bezeichnet: „Es muß sich rechnen, soll aber auch echte Verantwortung sein“ (Meckling 2004: 40). Ebenso jedoch wie eine Instrumentalisierung von Ethik als Mittel zum Zweck der nachhaltigen Gewinnsteigerung fragwürdig ist, muss auch die Forderung nach einer selbst auferlegten Begrenzung unternehmerischen Gewinnstrebens als realpolitisch fraglich betrachtet werden. Hier wird erneut offensichtlich, was bereits in der vorangegangenen Darstellung der Dimensionen von Bürgerschaft zutage getreten ist. Theoretische Inkonsistenzen und Grenzen der Übertragbarkeit des Bürgerbegriffs begleiten Konzeptionen der Konsumenten- und Unternehmensbürgerschaft. Dies gilt vor allem für Motive politischen Handelns, die nicht eindeutig entlang der Rollenskripte von Citoyen und Bourgeois verortet werden können. Auch wenn Entwürfe der Unternehmensbürgerschaft in stärkerem Maße eigenwohlorientierte Aspekte betonen, so sind Entwürfe der Konsumentenbürgerschaft ebenfalls mehrdimensional. Diese Abwendung von idealtypischen Vereinfachungen bürgerlicher Tugendvorstellung ist auch für empirische Fragen ausschlaggebend. Ein Ansatzpunkt ist hier die Bedeutung der Anerkennung durch das soziale Umfeld. Während die Forderung einer inneren moralischen Grundhaltung aufgrund des hohen Anspruchs an den einzelnen Bürger zu scheitern droht und eine unmittelbar gemeinwohlfördernde Dimension eines strategischen Handelns als fraglich zurückzuweisen ist, kann die Ausrichtung an kollektiv geltenden sozialen und politischen Normen durchaus als Ausdruck von Bürgertugend verstanden werden. Inwieweit, so gilt es zu untersuchen, entwickelt sich ein gesamtgesellschaftlicher Druck auf Konsumenten und Unternehmen, eine Rolle als ,good Consumer Citizen` bzw. good Corporate Citizen' auszufüllen?

\section{Fazit: Bürgerschaft als dynamischer Prozess der wechselseitigen Zuschreibung und Aushandlung}

Tabelle 2 fasst die identifizierten Spannungslinien sowie die daraus abgeleiteten Fragestellungen zusammen. Die Zusammenschau verdeutlicht zunächst die neuralgischen Punkte einer Übertragung des Bürgerbegriffs auf Konsumenten und Unternehmen. Aus der fehlenden institutionellen Verankerung resultieren Beschränkungen, was die Verbindlichkeit der mit einer Bürgerrolle verbundenen Rechte und Pflichten betrifft. In Bezug auf ,Regierungsansprüche' bestehen Grenzen der Übertragbarkeit des Bürgerbegriffs dadurch, dass die Machtausübung in politischen Arenen der Marktsphäre nur indirekt einer Legitimation durch (Wahl-)Verfahren unterliegt. Zudem setzt die mangelnde Gleichheit der Marktakteure einer Verwendung des Bürgerbegriffs in diesem Kontext Grenzen. 
Ausgestaltung der Bürgerrolle von Konsumenten und Unternehmen

\begin{tabular}{|c|c|}
\hline $\begin{array}{l}\text { Rechte und } \\
\text { Pflichten }\end{array}$ & $\begin{array}{l}\text { - Begrenzte institutionelle Einklagbarkeit von Bürgerrechten bzw. } \\
\text { Kontrolle der Einhaltung von Bürgerpflichten } \\
\text { - Selbstdefinition von Rechten und Pflichten } \\
\text { Etablierung eines Kanons von Rechten und Pflichten durch wechselseitige } \\
\text { Bequgnahme? }\end{array}$ \\
\hline $\begin{array}{l}\text { Regieren und } \\
\text { regiert } \\
\text { werden }\end{array}$ & $\begin{array}{l}\text { - Machtvorsprung von Unternehmen } \\
\text { - Gestaltung politischer Marktarenen zwischen Selbststeuerung und } \\
\text { Selbstbindung } \\
\text { Kontrolle durch kollektiviertes Konsumentenhandeln? }\end{array}$ \\
\hline $\begin{array}{l}\text { Freiheit und } \\
\text { Gleichheit }\end{array}$ & $\begin{array}{l}\text { - Ungleiche Handlungsspielräume durch ökonomische Ungleichheit } \\
\text { - Verknüpfung von Freiheits- und Gestaltungsansprüchen } \\
\text { Wablfreiheit als Ablehnung von Machtkontrolle (Unternebmen) } \\
\text { bzw. Widerspruch zu transnationaler Solidarität (Konsumenten)? }\end{array}$ \\
\hline $\begin{array}{l}\text { Bürgertugend } \\
\text { und } \\
\text { Gemeinwohl }\end{array}$ & $\begin{array}{l}\text { - Gemischte Motive als Sockel einer Bürgerrolle von Konsumenten und } \\
\text { - Tnternehmen } \\
\text { - Tugenddiskurs auf der Grundlage des Strebens nach Anerkennung } \\
\text { Vereinbarkeit universaler und partikularer Interessen? } \\
\text { Herstellung von Verbindlichkeit durch soziale und politische Normen? }\end{array}$ \\
\hline
\end{tabular}

Tabelle 2: Spannungslinien möglicher Bürgerrollen von Konsumenten und Unternehmen (eigene Darstellung)

Die vorangegangene konzeptionelle Analyse hat zudem konkrete empirische Fragestellungen aufgezeigt. So stellt sich durch die begrenzte institutionelle Einklagbarkeit von Bürgerrechten bzw. Kontrolle der Einhaltung von Bürgerpflichten zunächst die Frage, wie innerhalb des jeweiligen Bezugsrahmens Bürgerrechte und -pflichten durch Konsumenten und Unternehmen formuliert werden und durch welche Mechanismen diesen Geltung verschafft wird. Dabei gilt es vor allem zu untersuchen, inwieweit soziale und politische Normen bzw. Öffentliche Anerkennung oder Sanktion eine Kontrollfunktion ausfüllen können. Für beide Marktakteure stellt sich eine Bürgerrolle als ein Prozess der Selbst- und Fremdzuschreibung dar. In diesem Zusammenhang müssen vor allem die entstehenden Deutungskonflikte zwischen Consumer Citizen und Corporate Citizen in den Blick genommen werden. Deutungskonflikte sind dabei vor allem zu Aspekten der Machtausübung sowie hinsichtlich unterschiedlicher Vorstellungen des Gemeinwohls bzw. der Bürgertugend zu erwarten.

Trotz der skizzierten Probleme und Grenzen der Übertragbarkeit des Bürgerbegriffs stellt die Betrachtung von Konsumenten und Unternehmen in einer Bürgerrolle insgesamt eine Möglichkeit dar, politische Machtstrukturen in der Marktsphäre analytisch fassen zu können. Die vorgenommenen Systematisierungen sowie aufgeworfenen Forschungsfragen sollten hier als Ausgangspunkt betrachtet werden, um konkrete 
Konstellationen $\mathrm{zu}$ untersuchen, in denen Konsumenten und Unternehmen sich selbst eine Bürgerrolle zuschreiben bzw. ihnen eine Bürgerrolle zugeschrieben wird.

\section{Literaturverzeichnis}

Backhaus-Maul, H. (2004): Corporate Citizenship im deutschen Sozialstaat, in: Aus Politik und Zeitgeschichte, B 14, 23-30.

Backhaus-Maul, H. (2005): Corporate Citizenship. Liberale Gesellschaftspolitik als Unternehmensstrategie in den USA, in: Adloff, F./Birsl, U./Schwertmann, P. (Hrsg.): Wirtschaft und Zivilgesellschaft. Theoretische und empirische Perspektiven. Wiesbaden: VS Verlag für Sozialwissenschaften, 225-243.

Backhaus-Maul, H./Braun, S. (2007): Gesellschaftliches Engagement von Unternehmen in Deutschland. Konzeptionelle Überlegungen und empirische Befunde, in: Rote Seiten Stiftung \& Sponsoring, Jg. 10/Nr. 5, 1-15.

Backhaus-Maul, H./Schubert, I. (2005): Unternehmen und Konsumenten. Diffuse Verantwortung und schwache Interessen?, in: Forschungsjournal Neue Soziale Bewegungen, Jg. 18/Nr. 4, 78-88.

Bakan, J. (2005): Das Ende der Konzerne. Die selbstzerstörerische Kraft von Unternehmen. Leipzig: Europa Verlag.

Banerjee, S. B. (2008): Corporate Social Responsibility: The Good, the Bad and the Ugly, in: Critical Sociology, Vol. 34/No. 1, 51-79.

Barber, B. (2007): Consumed! Wie der Markt Kinder verführt, Erwachsene infantilisiert und die Demokratie untergräbt. München: C.H. Beck.

Baringhorst, S. (2008): Branded Political Communication. Euro-Asian Campaigns for Global Citizenship, in: Banarjee, I./Rao, M. (Hrsg.): Media and Development in Asia Regional Perspectives. Singapur: Asian Media Information and Communication Centre, 173-189.

Barnett, C./Cloke, P./Clarke, N./Malpass, A. (2005): Consuming Ethics: Articulating the Subjects and Spaces of Ethical Consumption, in: Antipode, Vol. 37/No. 1, 23-45.

Baxter, V. (2003): The Modern Corporate Accountability Movement: From Citizens' Power to Consumers' Power, in: Swords \& Plougshare: A Journal of International Affairs, Vol. 8/No. 1, 13-25.

Beetz, M. (2005): Leben zwischen Politik und Wirtschaft. Von der sanften Macht der Verbraucheröffentlichkeit, Online-Paper zur Tagung „Politisierter Konsum - konsumierte Politik“. Universität Gießen, 03.-04.06.2005, Link: www.politik-konsum.de/pdf/ verbraucher oeffentlichkeit.pdf (zuletzt abgerufen am 15.01.2008).

Behrent, M. (2003): Citizens in der Weltarena, in: Behrent, M./Wieland, J. (Hrsg.): Corporate Citizenship und strategische Unternehmenskommunikation in der Praxis, DNWESchriftenreihe Folge 11. München, Mering: Rainer Hampp, 21-35.

Bendell, J. (2000): Civil Regulation. A New Form of Democratic Governance for the Global Economy?, in: Bendell, J. (Hrsg.): Terms for Endearment. Business, NGOs and Sustainable Development. Sheffield: Greenleaf Publishing, 239-254.

Bevir, M./Trentmann, F. (2008): Civic Choices: Retrieving Perspectives on Rationality, Consumption and Citizenship, in: Soper, K./Trentmann, F. (Hrsg.): Citizenship and Consumption. London: Palgrave Macmillan, 19-33.

Biedermann, C. (2008): Corporate Citizenship als strategische Unternehmenskommunikation, in: Backhaus-Maul, H./Biedermann, C./Nährlich, S./Polterauer, J. (Hrsg.): Corporate Citizenship in Deutschland. Bilanz und Perspektiven. Wiesbaden: VS Verlag für Sozialwissenschaften, 291-306. 
Birch, D. (2001): Corporate Citizenship. Rethinking Business Beyond Corporate Social Responsibility, in: Andriof, J./McIntosh, M. (Hrsg.): Perspectives on Corporate Citizenship. Sheffield: Greenleaf Publishing, 53-65.

Carter, N./Huby, M. (2005): Ecological Citizenship and Ethical Investment, in: Environmental Politics, Vol. 14/No. 2, 255-272.

Clarke, N./Barnett, C./Cloke, P./Malpass, A. (2007): The Political Rationalities of Fair-Trade Consumption in the United Kingdom, in: Politics \& Society, Vol. 35/No. 4, 583-607.

Cohen, L. (2003): A Consumers' Republic. The Politics of Mass Consumption in Postwar America. New York: Vintage.

Crane, A./Matten, D./Moon, J. (2008): Corporations and Citizenship. Cambridge: Cambridge University Press.

Crane, A./Matten, D./Moon, J. (2004): Stakeholders as Citizens? Rethinking Rights, Participation, and Democracy, in: Journal of Business Ethics, Vol. 53/No. 1-2, 107-122.

Crouch, C. (2004): Post-Democracy. Cambridge, Malden: Polity Press.

Dickinson, R. (1996): Consumer Citizenship. The United States, in: Business in the Contemporary World, Vol. 8/No. 3-4, 255-273.

Dickinson, R./Carsky, M. (2005): The Consumer as Economic Voter, in: Harrison, R./Newholm, T./Shaw, D. (Hrsg.): The Ethical Consumer. London: Sage, 25-36.

Gabriel, Y./Lang, T. (2006): The unmanageable consumer. London: Sage.

Gonin, M. (2006): Die Trennung des Unternehmens vom Bürger - eine historisch-kritische Perspektive, in: Patry, Eric/Ulrich, Peter (Hrsg.): Marktwirtschaft als Teil einer bürgergesellschaftlichen Gesamtordnung. St. Gallen: Institut für Wirtschaftsethik der Universität St. Gallen, 41-56.

Guay, T./Doh, J./Sinclair, G. (2004): Non-governmental Organizations, Shareholder Activism, and Socially Responsible Investments. Ethical, Strategic, and Governance Implications, in: Joumal of Business Ethics, Vol. 52/No. 1, 125-139.

Habisch, A. (2003): Corporate Citizenship. Gesellschaftliches Engagement von Unternehmen in Deutschland. Berlin, Heidelberg: Springer.

Habisch, A./Schwary, C. (2012): CSR als Investition in Human- und Sozialkapital, in: Schneider, A./Schmidpeter, R. (Hrsg.): Corporate Social Responsibility. Verantwortungsvolle Unternehmensführung in Theorie und Praxis. Berlin, Heidelberg: Springer Gabler, 113-133.

Henberger, F./Oppen, M./Reimer, S. (2004): Der deutsche Weg zum bürgerschaftlichen Engagement von Unternehmen. Reihe ,betrifft: Bürgergesellschaft' Nr. 12. Bonn: FriedrichEbert-Stiftung.

Holzer, B. (2006): Political Consumerism between Individual Choice and Collective Action: Social Movements, Role Mobilization and Signalling, in: International Journal of Consumer Studies, Vol. 30/No. 5, 405-415.

Janes, J./Stucbtey, T. (2008): Making Money by Doing Good, in: Aus Politik und Zeitgeschichte, 31/2008, 20-25.

Kneip, V. (2010): Consumer Citizenship und Corporate Citizenship. Bürgerschaft als politische Dimension des Marktes. Baden-Baden: Nomos.

Lamla, J. (2005): Kontexte der Politisierung des Konsums. Die Zivilgesellschaft in der gegenwärtigen Krisenkonstellation von Politik, Ökonomie und Kultur, Online-Paper zur Tagung „Politisierter Konsum - konsumierte Politik“. Universität Gießen, 03.-04.06.2005, Link: www.politik-konsum.de/pdf/lamla_kontexte.pdf (zuletzt abgerufen am 10.01.2008).

Lamla, J. (2008): Consumer Citizen: The Constitution of Consumer Democracy in Sociological Perspective, in: German Policy Studies, Vol. 4/No. 1, 131-166. 
Lee, S./Baik, Y. (2010): Corporate Lobbying in Antidumping Cases: Looking into the Continued Dumping and Subsidy Offset Act, in: Journal of Business Ethics, Vol. 96/No. 3, 467-478.

Lin, C. (2010): Modeling Corporate Citizenship, Organizational Trust, and Work Engagement Based on Attachment Theory, in: Journal of Business Ethics, Vol. 94/No. 4, 517-531.

Linton, A. (2003): Introduction, in: Politics \& Society, Vol. 31/No. 3, 359-362.

Lipschutr, R. (2003): Regulation for the Rest of Us? Global Social Activism, Corporate Citizenship, and the Disappearance of the Political, Link: http://repositories.cdlib.org/cgirs/ CGIRS-2003-1 (zuletzt abgerufen am 01.10.2009).

Littler, J. (2009): Radical Consumption. Shopping for Change in Contemporary Culture. Berkshire: Open University Press.

Maak, T. (1999): Die Wirtschaft der Bürgergesellschaft. Ethisch-politische Grundlagen einer Wirtschaftspraxis selbstbestimmter Bürger. Bern, Stuttgart, Wien: Verlag Peter Haupt.

Marsden, C./Andriof, J. (1998): Towards an Understanding of Corporate Citizenship and How to Influence it, in: Citizenship Studies, Jg. 2/ Vol. 2, 329-352.

Matten, D./Crane, A. (2005): Corporate Citizenship: Toward an Extended Theoretical Conceptualization, in: Academy of Management Review, Vol. 30/No. 1, 166-179.

Matten, D./Crane, A./Chapple, W. (2003): Behind the Mask: Revealing the True Face of Corporate Citizenship, in: Journal of Business Ethics, 45/No. 1-2, 109-120.

McIntosh, M./Leipriger, D./Jones, K./Coleman, G. (1998): Corporate Citizenship. Successful Strategies for Responsible Companies. London: Financial Times/Pitman.

Meckling, J. (2004): Netzwerkgovernance - Corporate Citizenship und Global Governance. Münster: Lit.

Messner, D. (1998): Architektur der Weltordnung. Strategien zur Lösung globaler Probleme, in: Internationale Politik, 11/1998, 17-24.

Micheletti, M. (2003): Political Virtue and Shopping. Individuals, Consumerism, and Collective Action. New York: Palgrave Macmillan.

Micheletti, M. (2004): Just Clothes? Discursive Political Consumerism and Political Participation. Paper zum European Consortium for Political Research, Workshop „Emerging Repertoires of Political Action“. Uppsala, 14.-18.04.2004.

Micheletti, M./Stolle, D. (2007): Mobilizing Consumers to Take Responsibility for Global Social Justice, in: The ANNALS of the American Academy of Political and Social Science, No. 611, 157-175.

Mirvis, P./Googins, B. (2009): Moving to Next Generation Corporate Citizenship, in: CCC Debatte, 01.

Moon, J./Crane, A./Matten, D. (2008): Citizenship als Bezugsrahmen für politische Macht und Verantwortung der Unternehmen, in: Backhaus-Maul, H./Biedermann, C./Nährlich, S./Polterauer, J. (Hrsg.): Corporate Citizenship in Deutschland. Bilanz und Perspektiven. Wiesbaden: VS Verlag für Sozialwissenschaften, 45-67.

Näbrlich, S. (2008): Euphorie des Aufbruchs und Suche nach gesellschaftlicher Wirkung, in: Aus Politik und Zeitgeschichte, 31/2008, 26-31.

Oldfield, A. (1998): Citizenship: An Unnatural Practice? In: Turner, B./Hamilton, P. (Hrsg.): Citizenship. Critical Concepts, Band 1. London, New York: Routledge, 188-198.

Oliviero, M./Simmons, A. (2002): Who's Minding the Store? Global Civil Society and Corporate Responsibility, in: Glasius, M./Kaldor, M./Anheier, H. (Hrsg.): Global Civil Society 2002, Oxford: Oxford University Press, 77-108.

Paar, S. (2005): Die Kommunikation von Corporate Citizenship. Bamberg: Difo-Druck $\mathrm{GmbH}$. 
Palacios, J. (2004): Corporate Citizenship and Social Responsibility in a Globalized World, in: Citizenship Studies, Vol. 8/No. 4, 383-402.

Polterauer, J. (2005): Corporate Citizenship. Systemfunktionalistische Perspektiven, in: Adloff, F./Birsl, U./Schwertmann, P. (Hrsg.): Wirtschaft und Zivilgesellschaft. Theoretische und empirische Perspektiven. Wiesbaden: VS Verlag für Sozialwissenschaften, 97-126.

Reich, R. (2008): Superkapitalismus. Wie die Wirtschaft unsere Demokratie untergräbt. Frankfurt a. M.: Campus.

Reimer, S. (2004): Corporate Citizenship in Diskussion und Praxis, in: Reimer, S./Wettenmann, T./Backhaus-Maul, H. (Hrsg.): Aktuelle Beiträge zu Corporate Citizenship. Diskussionspapiere zum Nonprofit-Sektor Nr. 26, Link: www.aktive-buergerschaft.de/vab/ resourcen/ diskussionspapiere/wp-band26.pdf (zuletzt abgerufen am 14.07.2007).

Riedel, M. (1972): Bürger, Staatsbürger, Bürgertum, in: Brunner, O./Conze, W./Koselleck, R. (Hrsg.): Geschichtliche Grundbegriffe. Historisches Lexikon zur politisch-sozialen Sprache in Deutschland. Stuttgart: Ernst Klett Verlag, 672-725.

Rieger, G. (1998): Citoyen, in: Nohlen, D./Schultze, R./Schüttemeyer, S. (Hrsg.): Lexikon der Politik. Band 7: Politische Begriffe. München: C.H. Beck, 94-95.

Rondinelli, D. (2002): Transnational Corporations: International Citizens or New Sovereigns? In: Business and Society Review, Vol. 107/No. 4, 391-413.

Rose, N. (1999): Powers of Freedom. Reframing Political Thought. Cambridge: Cambridge University Press.

Rosenkrands, J. (2004): Policizing Homo Economicus. Analysis of Anti-Corporate Websites, in: Donk, W./Loader, B./Nixon, P./Rucht, D. (Hrsg.): Cyberprotest. New Media, Citizens and Social Movements. London, New York: Routledge, 57-76.

Sack, D. (2005): Profession, Partnerschaft und doppelter Gewinn. Zur Gouvernementalität von Corporate Citizenship, in: Adloff, F./Birsl, U./Schwertmann, P. (Hrsg.): Wirtschaft und Zivilgesellschaft. Theoretische und empirische Perspektiven. Wiesbaden: VS Verlag für Sozialwissenschaften, 175-198.

Scammell, M. (2000): The Internet and Civic Engagement. The Age of the Citizen Consumer, in: Political Communication, Vol. 17/No. 4, 351-355.

Schawalder, A. (2006): Die Wirtschaft in der Bürgergesellschaft - das Grundverständnis aus kapitalistisch-libertärer und republikanisch-liberaler Perspektive, in: Patry, E./Ulrich, P. (Hrsg.): Marktwirtschaft als Teil einer bürgergesellschaftlichen Gesamtordnung. St.Gallen: Institut für Wirtschaftsethik der Universität St.Gallen, 5-21.

Schoenheit, I. (2007): Politischer Konsum. Ein Beitrag zum faustischen Konsumentenverhalten, in: Jäckel, Michael (Hrsg.): Ambivalenzen des Konsums und der werblichen Kommunikation. Wiesbaden: VS Verlag für Sozialwissenschaften, 211-234.

Schrader, U. (2003): Corporate Citizenship: Die Unternehmung als guter Bürger? Berlin: Logos.

Schwertmann, P. (2005): Unternehmensstiftungen im Spannungsfeld von Eigennutz und Gemeinwohl, in: Adloff, F./Birsl, U./Schwertmann, P. (Hrsg.): Wirtschaft und Zivilgesellschaft. Theoretische und empirische Perspektiven. Wiesbaden: VS Verlag für Sozialwissenschaften, 199-223.

Seitz, B. (2002): Corporate Citizenship. Rechte und Pflichten der Unternehmung im Zeitalter der Globalität. Wiesbaden: Gabler.

Soper, K. (2008): 'Alternative Hedonism' and the Citizen-Consumer, in: Soper, K./Trentmann, F. (Hrsg.): Citizenship and Consumption. London: Palgrave Macmillan, 191-205.

Sorensen, M. (2005): Liberal Democratic Society and Political Consumerism - a Problematic Combination, in: Boström, M./Follesdal, A./Klintman, M./Micheletti, M./Sorensen, M. (Hrsg.): Political Consumerism: Its Motivations, Power, and Conditions in the Nordic Countries and Elsewhere. Kopenhagen: TemaNord, 59-76. 
Spaargaren, G./Martens, S. (2005): Globalisation and the Role of Citizen-Consumers in Environmental Politics, in: Boström, M./Follesdal, A./Klintman, M./Micheletti, M./Sorensen, M. (Hrsg.): Political Consumerism: Its Motivations, Power, and Conditions in the Nordic Countries and Elsewhere. Kopenhagen: TemaNord, 25-58.

Stolle, D./Micheletti, M. (2005): Warum werden Käufer zu ,politischen Verbrauchern??, in: Forschungsjournal Neue Soziale Bewegungen, Jg. 18/Nr. 4, 41-52.

Svendsen, G. (2011): Evaluating and Regulating the Impacts of Lobbying in the EU?, in: Environmental Policy \& Governance, Vol. 21/No. 2, 131-142.

Thompson, G. (2005): Global Corporate Citizenship. What Does it Mean?, in: Competition and Change, Vol. 9/No. 2, 131-152.

Tichy, N./Mc Gill, A./St. Clair, L. (Hrsg.) (1997): Corporate Global Citizenship. Doing Business in the Public Eye. San Francisco: New Lexington Press.

Ulrich, P. (2000): Republikanischer Liberalismus und Corporate Citizenship. Von der ökonomistischen Gemeinwohlfiktion zur republikanisch-ethischen Selbstbindung wirtschaftlicher Akteure, Berichte des Instituts für Wirtschaftsethik 88. St. Gallen: Institut für Wirtschaftsethik der Hochschule St. Gallen.

Ulrich, P. (2005): Zivilisierte Marktwirtschaft. Wirtschaftsbürgerrechte als sozioökonomische Voraussetzung einer voll entfalteten Bürgergesellschaft, in: Ethica, Jg. 13/Nr. 1, 13-34.

Westebbe, A./Logan, D. (1995): Corporate Citizenship. Unternehmen im gesellschaftlichen Dialog. Wiesbaden: Gabler.

Wieland, J. (2003): Corporate Citizenship, in: Behrent, M./Wieland, J. (Hrsg.): Corporate Citizenship und strategische Unternehmenskommunikation in der Praxis, DNWESchriftenreihe Folge 11. München, Mering: Rainer Hampp, 13-19.

Willmott, M. (2003): Citizen brands. Corporate citizenship, trust and branding, in: The Journal of Brand Management, Vol. 10/No. 4-5, 362-369.

Young, I. (1995): Polity and Group Difference: A Critique of the Ideal of Universal Citizenship, in: Beiner, Ronald (Hrsg.): Theorizing Citizenship. New York: State University of New York Press, 175-207.

Zadek, S. (2002): Third Generation Corporate Citizenship. Foreign Policy Centre: London.

Zadek, S. (2007): The Civil Corporation. The New Economy of Corporate Citizenship. Earthscan: London.

Zadek, S./Amalric, F. (1998): Consumer Works!, in: Development: Journal of the Society for International Development, Vol. 41/No. 1, 7-14. 